\title{
Physical and Chemical Characterization of Volcanic Dust Particles from Mt. Semeru and Mt. Bromo, and the Research of Their Influences on the Productivity of Nearby Agricultural Lands
}

\author{
Alamsyah M. Juwono
}

\begin{abstract}
A study to, physically and chemically, characterise the volcanic dust particles, from Mt. Semeru and Mt. Bromo, has been done. All samples were treated and prepared for examination using three different equipment, XRD, FE-SEM, and $X R F$. The results from analysis using XRF indicated that, both sample clusters have some common chemical atomic/molecular contents, and both are dominated by the Fe and Si traces. However, the results from analysis using other two equipment do not show the same dominance of Fe and Si. XRD results have indirectly indicated the present and dominance of $\mathrm{Si}$ and $\mathrm{Fe}$. The main contents of the samples from Mt Semeru were $\mathrm{Fe}(39.2 \%), \mathrm{Si}(27.9 \%)$, and other elements such as $\mathrm{Ca}(12,7 \%)$, Al (12), and $K(2,69 \%)$. Zinc (Zn) was an element with lowest concentration, $(0.03 \%)$. Results from the use of XRD, on the samples from Mt. Bromo, indicated the close similarity of their spectral patterns with those of Semeru. Elements, such as Ca, Al and $K$, exist in the both sample clusters. Comparing the current study, with study of Omar [8], suggested that Si found in the sample Sem1, for instance, has a crystalline structure, with Miller index of [ [ 11 1], and 2-theta angle of about $28^{\circ}$, while the

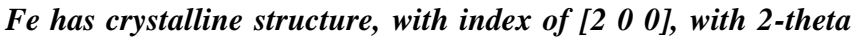
angle of about $65^{\circ}$. In general, the study using the three kind of equipment for samples from the two mountains, indicate the existence of many metal traces, mostly transition metals, with their various concentrations, i.e., $\mathrm{Fe}, \mathrm{Na}, \mathrm{Ca}, \mathrm{Mg}, \mathrm{Ti}, \mathrm{K}, \mathrm{Mn}, \mathrm{Cu}$, $\mathrm{V}, \mathrm{Cr}, \mathrm{Rb}, \mathrm{Sr}, \mathrm{Ba}, \mathrm{Zn}$, and Pb. Toxic traces such as $\mathrm{F}$ was not detected. From FE-SEM study it was revealed, that, the volcanic dust particles, have sizes of order of ten to few hundreds of micrometer
\end{abstract}

Keywords: Volcanic dust, Semeru, Bromo, particle size, chemical elements, carbon, oxygen, silicon, iron, metal traces, transition metals.

\section{INTRODUCTION}

As one of the countries located in the ring of fire zone, Indonesia has hundreds of volcanoes, both active and non-active, throughout the country. Therefore, volcanic eruptions are common events. The community and the government have had a long experience, in dealing with volcanic eruptions, fire, and all other consequences.

Revised Manuscript Received on January 10, 2020.

Alamsyah M. Juwono, lecture, Study Program of Geophysics Engineering, Deparment of Physics, Faculty of Mathematics and Sciences, Brawijaya University. Indonesia
Mt. Bromo and Mt. Semeru, in East Java, are active volcanoes. Mt. Semeru has an interesting eruption character, that is, small phreatic eruption with relatively high frequency, with the material upthrow reaching a height of only about 200 to $300 \mathrm{~m}$, above the mountain peak.

Mt. Bromo, on the other hand, has a pattern of eruption that is similar to the pattern of eruptions in general volcanoes in Indonesia. Mt. Bromo had become one of the main tourist destinations, and, therefore, an eruption that occurs could have an extraordinary impact

The spread of these volcanoes have caused health problems in the community, in a wide range of areas, also brought losses in agriculture, as well as in transportation.

Losses in agriculture, for example, were the decrease of fruit production, especially apples, in nearby areas, such as in the Poncokusumo District. Impact on the public health has received little, or moderate attention, although it may cause emergence of respiratory diseases, and eye irritation, due to poor air quality in nearby residential areas. Losses in the economic can be derived from the decline in population activities in the affected locations, such as in tourist visits.

The minerals contained in volcanic ash depend on the chemical material contained in the magma that forms it, and, is always dominated by the element silica, accompanied by other metals, such as iron (Fe) and magnesium (Mg). Silicate content in felsic volcanic ash can reach $70 \%$. Witham et al [15] reported that from observation of fresh volcanic ash, more than 55 different types of ions were obtained, the main ones being $\mathrm{Na}^{+}, \mathrm{K}^{+}, \mathrm{Ca}^{2+}$, and $\mathrm{Mg}^{2+}$ and $\mathrm{Cl}^{-}$, and $\mathrm{F}^{-}$, and $\mathrm{SO}^{2+}{ }_{4}$.

Ash was once thought to be a noble material (inert), and difficult to react with other materials, and does not have a negative impact on health. However, there is evidence that particles from volcanic ash can be part of air and water pollution, which affects health [5]. The particles of fresh volcanic ash have a surface that has not been weathered, and has not been oxidized, and can therefore carry non-volatile materials of acid molecules, and metals. Such particles tend to be spherical in shape, with a diameter of $<100 \mu \mathrm{m}$ in diameter. Some may be electrically charged [2].

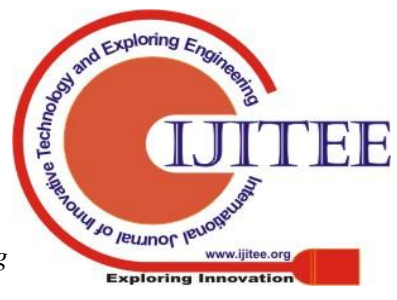


Physical and Chemical Characterization of Volcanic Dust Particles from Mt. Semeru and Mt. Bromo, and the Research of Their Influences on the Productivity of Nearby Agricultural Lands

The impacts caused by volcanic eruption activities, especially those related to volcanic ash emission, can be in the form of material losses, as well as loss of life. Material losses caused by volcanic eruptions can be in the form of losses in the form of damage of facilities, damage of plants, water resources, damage to transportation equipment, and disruption of land, sea and especially air transportation. While the non-material losses can be in the form of death due to disruption of the respiratory system, ash particle poisoning, etc. related to volcanic ash.

The fall of volcanic ash on agricultural land, will cause disruption to soil fertility. According to Nanzyo, et al. [7], immediately after the fall of volcanic ash, then the surface on the local land will form a layer of soil, called Andisol. Andisol soil makes local land has different properties from the normal soil. Andisol soils are generally infertile, and tend to make plants not grow well, due to the low soil nutrient content of plants, and due to the present of toxic elements [11]. Only after experiencing washing by rain water, in a relatively long period of time, the state of the land can be restored to normal

\section{MATERIALS AND METHODS}

This study is investigating chemical mater contents, and physical properties, of volcanic dust particles. Samples were collected from surrounding of Mt. Semeru and Mt. Bromo, during months of September to November 2019. The samples were treated, by to have them cleaned up from non-dust materials, and heated up to $500^{\circ} \mathrm{C}$, for 30 minutes. The heating was aimed to make the samples dry enough, so that they do not contain water. The samples were also filtered up to 50 mesh, to sort them, so that the maximum size of dust particle is about $0.3 \mathrm{~mm}$. The filtered samples were further crushed to make them finer.

We conducted analysis of the samples, by using three different equipment, that's, the XRF, XRD, and FE-SEM. We selected 7 samples from Mt. Bromo, and named them as Bro1, Bro2, etc; and 5 samples from Mt. Semeru, and named them as Sem1, Sem2, etc., accordingly.

The analysis using XRF were conducted at Central Lab of State University of Malang, while the analysis using XRD and FE-SEM were both conducted at the Central Lab of Brawijaya University. The results of the analysis are presented in the following

\section{A. Analysis Using XRF}

Results of the analysis using XRF, were only obtained for two samples from each sample cluster, that's, Sem1 and Sem3 samples, from Mt. Semeru, and Bro2 and Bro4 samples, from Mt. Bromo. The typical results of the analysis are as following.

Fig. 1 displays result of the test using XRF, for which, it indicates the spectrum of the particles' count per-dispersive-energy unit (a), and various chemical elements, and their corresponding concentrations (b). It is shown in the Fig. 1 (b), that, in the sample Sem1, the most dominant element is Iron (Fe), with a concentration of $39.2 \%$, followed by silicon ( $\mathrm{Si}$ ) with a concentration of
27.9\%. Other elements that are classified as dominant are $\mathrm{Ca}$ (12.7\%), $\mathrm{Al}(12)$, and $\mathrm{K}(2.69 \%)$. The Zinc ( $\mathrm{Zn})$ element is the lowest concentration element, detected in the sample.

Samples from Bromo, which were represented by Bro2 and Bro4, under analysis using XRF produced similar results, with those result from Sem1. The dominance of the element traces is also similar, that's, Fe (44.44\%), Si (24.9\%), $\mathrm{Al}(15 \%)$, and $\mathrm{Ca}(8 \%)$. The $\mathrm{Zn}$ was also detected with lowest concentration.

The overall results of analysis using XRF, indicated, that, basically, chemical contents of samples from Mt. Semeru and Mt. Bromo are the similar. The similarity is clarified by the similar trends of concentration lines, as presented in Fig. 2.

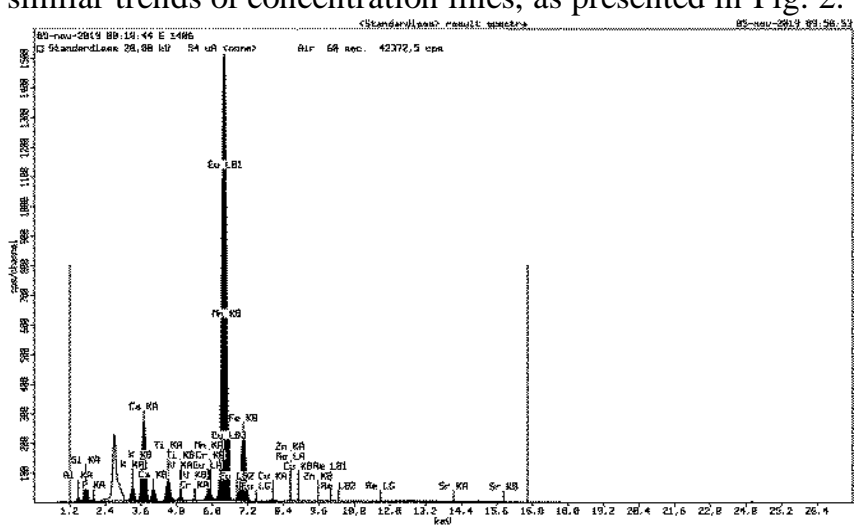

(a)

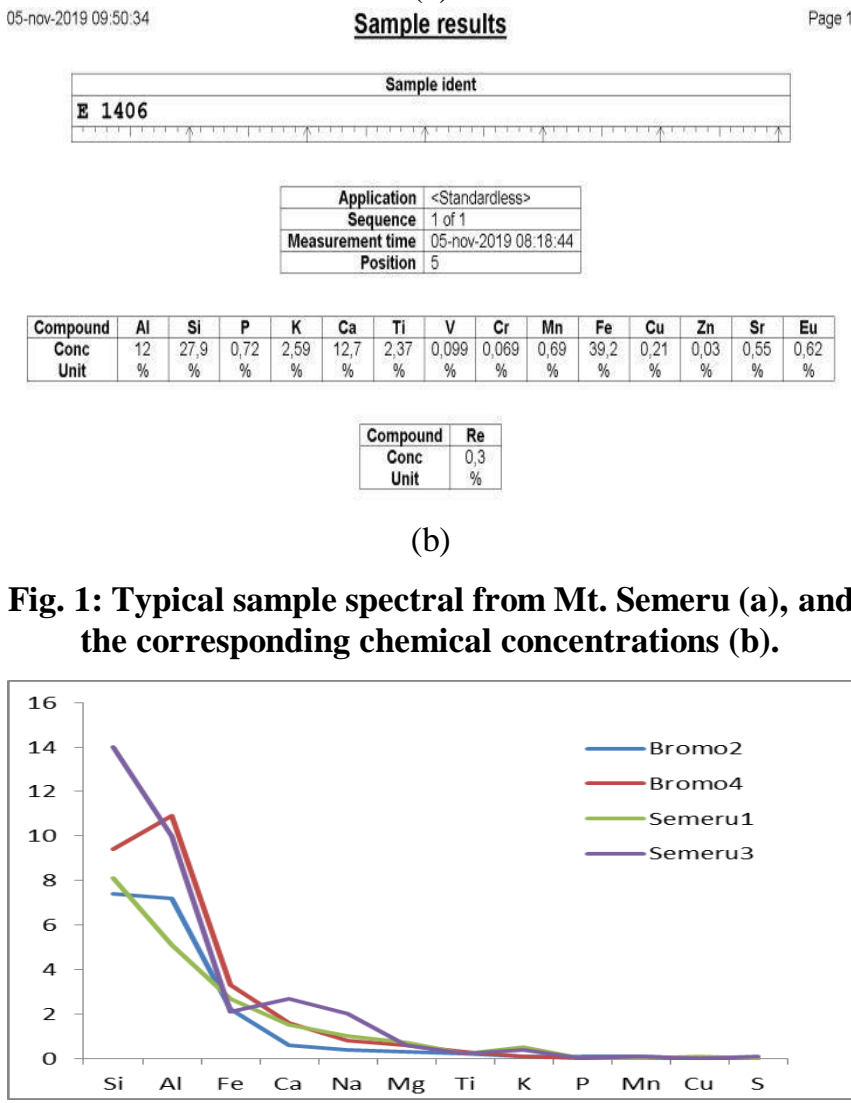

Fig. 2: Concentrations of various elements from samples of Sem1, Sem3, Bro2, and Bro4. The trends of concentration lines indicate the similarity the contents and their corresponding concentrations, from both sample clusters 


\section{B. Analysis Using XRD and FE-SEM}

Typical results of the test using XRD, for samples from Bromo, all are combined, is indicated by Fig. 3(a), while those of combination of samples of Sem1, Sem3, Sem5, Sem7, and Sem8, is indicated in the Fig. 3(b). It should be noted, that the horizontal axis in these figures is 2-theta angle, which, is angle between incident and diffracted beams, in the XRD engine. In these figures, the spectra of all samples look similar.

There is one distinct peak that is present in all spectra, which is not explicitly identified, which the element the peaks correspond to.

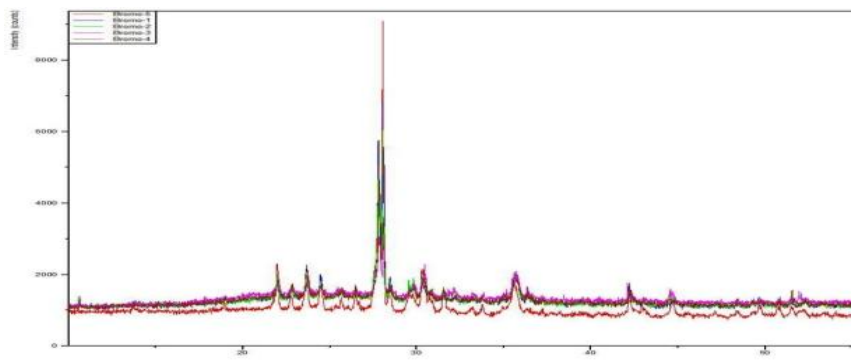

(a)

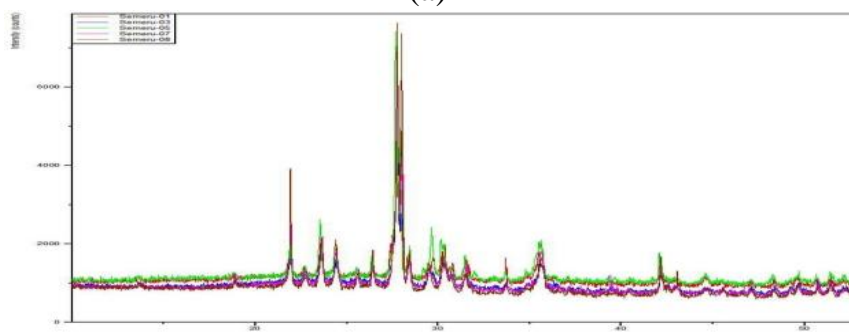

(b)

Fig. 3: Spectra of samples from two sample clusters, Bro1 to Bro5 (a), and Sem1, Sem3, Sem5, Sem7, and Sem8 (b).

The spectra look very much the same, which indicate similarity between contents in the two sample clusters

Further similarity is also justified by spectra of combination of samples Sem1, Sem3, Bro2, and Bro4, as indicated in the Fig. 4, where the four samples are plotted together. As can be seen in the Fig. 4, the spectra of the four samples look very much the same, which indicate that the elemental content of the samples, and their corresponding concentrations are basically the same.

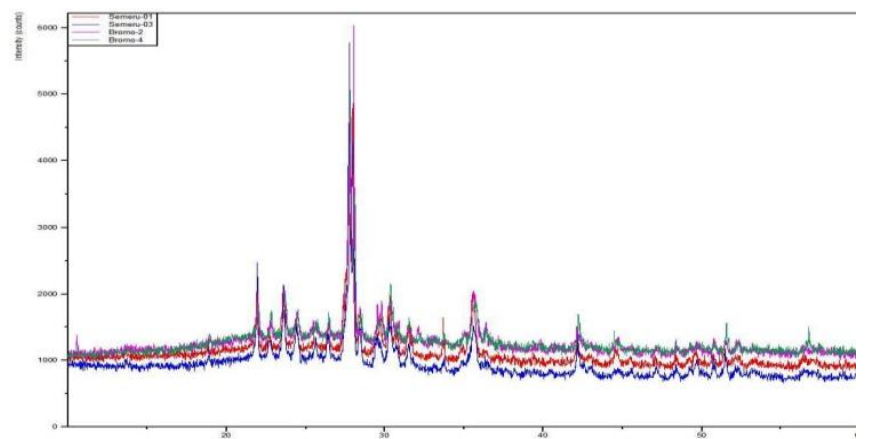

Fig. 4: Spectra of combination of Sem1, Sem3, Bro2, and Bro4

The main peak, as indicated in the Fig. 3 (a) and (b), and Fig. 4, was interpreted by the help of the work of Omar [8], which indicated that such peak, at 2-theta angle of about $28^{\circ}$,

is due to element of $\mathrm{Si}$. Omar [8] also indicated, that, the peak which appears in the 2-theta angle of about $65^{\circ}$, is due to element of Fe. Closer look at the Fig. 3 (b), such peak indeed exists in the spectrum of sample of Sem5.

Typical result of analyzing samples using FE-SEM is shown in Fig. 5. This spectrum is from sample Sem1, which clearly indicates the count per-dispersive energy unit, which represents the relative concentration of each element. Not like the other two equipment, whose results have been discussed earlier, the FE-SEM also result displays the present of the oxygen, and carbon (C). However, the FE-SEM results indicate the concentrations of the each element quite differently in their values. This may be due to the detection of Oxygen, which was not detected, and hence, is not counted in the percentage in the other two equipment.

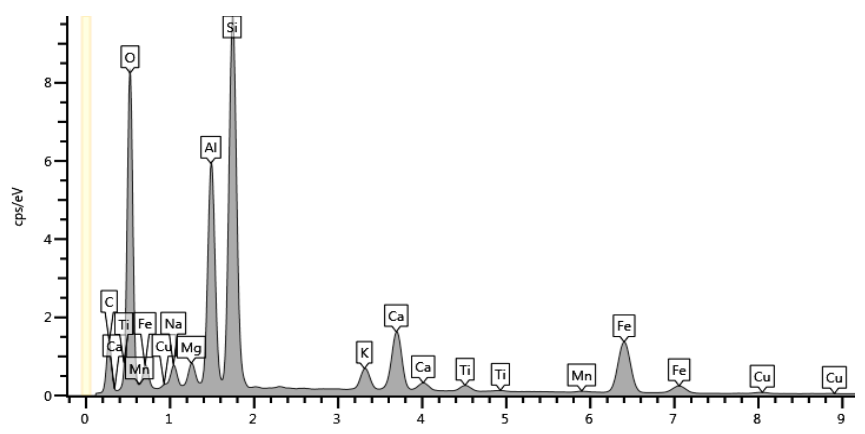

Fig. 5: Spectrum of chemical element content of sample Sem1

Some data examples, which display the difference between result from XRF test and FE-SEM test, are given in the Table 1. From the table, it can be seen, that, while FE-SEM detected oxygen and carbon, the XRF did not. Main elements, like $\mathrm{Si}, \mathrm{Fe}$ and $\mathrm{Al}$, were detected with low concentration in the FE-SEM Test, and were detected with a lot higher concentrations in the XRF Test. The large discrepancy might come from the fact, that, in the XRD Test, there are two elements which are not present in the XRD Test. Oxygen is not intrinsic constituent of volcanic dust particle. In the FE-SEM Test, it was detected with high concentration, that makes the concentrations of other elements become relatively low.

Table 1: Comparison of Elemental Results from FE-SEM Test and from XRF Test

FE-SEM

$\mathrm{XRF}$

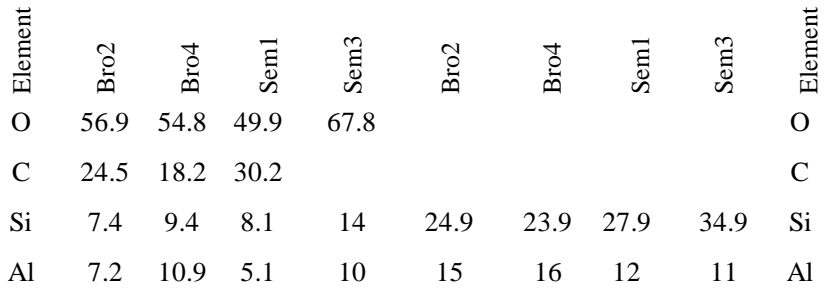


Physical and Chemical Characterization of Volcanic Dust Particles from Mt. Semeru and Mt. Bromo, and the Research of Their Influences on the Productivity of Nearby Agricultural Lands

$\begin{array}{lllllccccc}\mathrm{Fe} & 2.2 & 3.3 & 2.7 & 2.1 & 44.44 & 46.59 & 39.2 & 30.6 & \mathrm{Fe} \\ \mathrm{Ca} & 0.6 & 1.6 & 1.5 & 2.7 & 8.01 & 6.17 & 12.7 & 15 & \mathrm{Ca}\end{array}$

The results from FE-SEM Test, i.e., the photographs show the physical forms of the sample particle. For example, Fig.6 shows the micrograph of Bro2 sample. The photo shows that volcanic dust particles have irregular form, with jugged sharp edge, with length sizes ranging from tens to few hundreds of micrometer.

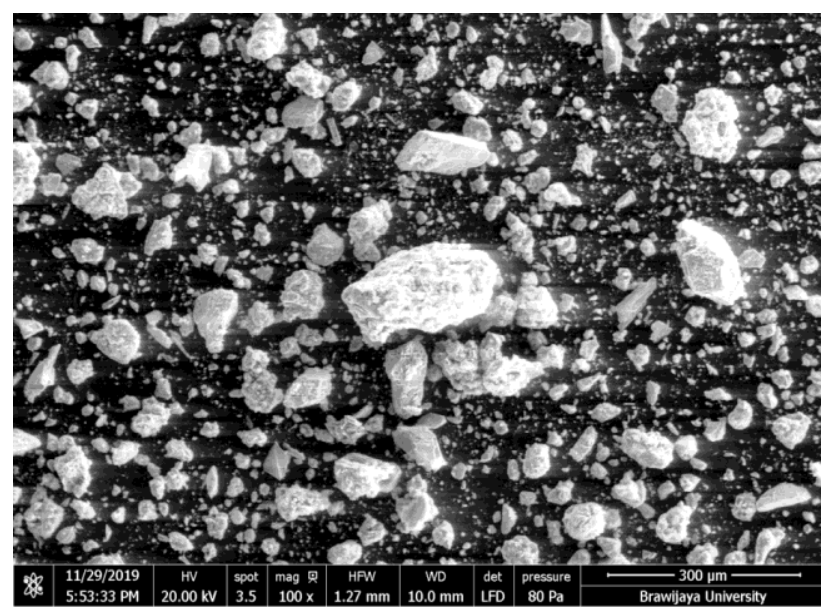

Fig. 6: Micrograph of the Bro2 sample, which shows volcanic dust particles contained in the sample. Particle builds are generally irregular, of varying lengths, with size estimates ranging from tens to several hundred micrometers.

From various results obtained, as discussed above, the trace of halogenic elements, such as fluorine, which is a toxic chemical, was not detected. This may be due, that, the samples that we worked on, were not fresh, but have been deposited on the ground for few months, for samples from Mt. Bromo, and for about 5 years, for samples from Mt. Semeru, from the last times when these mountains erupted. Therefore, the volcanic dust samples used in this study, may have been washed by rain water again and again, and lost some chemical traces.

\section{RESULTS AND DISCUSSION}

From what have been explained above, it can be said, that, in general, the chemical composition of samples from the two sample clusters, corresponding to the two mountains, are very much similar. Analysis that has been done produced results that indicate the present of various metal traces that contained in the samples, with similar concentrations.

The main elements, which are detected from the three types of sample tests are: Silicon (Si), Aluminum (Al), Iron $(\mathrm{Fe})$, Calcium $(\mathrm{Ca})$. The concentration of $\mathrm{Si}$ and $\mathrm{Fe}$ from the FESEM-EDS Test and the XRF Test showed a large difference, where in the later test, the two elements were detected with high concentrations. The present of $\mathrm{Si}$ with high concentrations was also shown by the results of the XRD-Test, where $\mathrm{Si}$ with high concentrations presents at 2-point angle of about $27^{\circ}-28^{\circ}$. XRD Test also shows that the contents of $\mathrm{Fe}$ in both groups of samples are of moderate concentrations. Comparison of the results of the XRF-Test with the results of research [1] [13], shows, that, the results of the XRF-Test are more complete results.

As shown in Table 1, where comparison is made for the results from FE-SEM and XRF Tests, although in most cases both tests detected the same elements, but their corresponding concentration could be very much different. The Si averaged concentration from FE-SEM is $9.7 \%$, while those from XRF test have average value of $27.9 \%$, that is about three times larger. For Fe, element was detected with average concentration of $2.6 \%$ in FE-SEM test, while it was detected in XRF test with concentration of $40 \%$. The concentrations of $\mathrm{Al}$, from both tests also indicate obvious difference, i.e., $8.3 \%$ for FE-SEM test, and $13.5 \%$ for XRF test. The $\mathrm{K}$ also shows clear difference, i.e., $0.28 \%$ for FE-SEM and $2.07 \%$ for XRF.

Overall, the element that contained in the samples are metal traces from transition metal group, i.e., $\mathrm{Fe}, \mathrm{Na}, \mathrm{Ca}$, $\mathrm{Mg}, \mathrm{Ti}, \mathrm{K}, \mathrm{Mn}, \mathrm{Cu}, \mathrm{V}, \mathrm{Cr}, \mathrm{Rb}, \mathrm{Sr}, \mathrm{Ba}$, and $\mathrm{Pb}$.

Traces of halogenic elements, such as fluorine, which is a toxic chemical, was not detected. This may be due, that, the samples that we worked on, were not fresh, but have been deposited on the ground for few months, for samples from Mt. Bromo, and for about 5 years, for samples from Mt. Semeru, if times from the last times when these mountains erupted. Therefore, the volcanic dust samples used in this study, may have been washed by rain water again and again, and lost some chemical traces. Fluorine and other elements from halogen group in the periodic table are considered as toxic elements, which, may cause damage to the plant lives. It may explain why most soils seem to be not fertile, often followed by the death of plants, when they have just poured by dust, or ash, from mountain eruption. After washed by rain, the soils would be back to normal condition.

The FE-SEM revealed that the size of the dust particle are mostly tens up to few hundreds micrometer, with irregular forms.

\section{CONCLUSION}

From what we have done, in investigating the physical and chemical characteristics of the volcanic dust particle, it can be concluded that:

1. In general, volcanic dust samples from Mt. Bromo and Mt. Semeru have similar chemical and physical characteristics, both in terms of the main constituent elements, as well as their physical appearance and sizes

2. Overall, the element that contained in the samples are metal traces from transition metal group, i.e., $\mathrm{Fe}, \mathrm{Na}, \mathrm{Ca}$, $\mathrm{Mg}, \mathrm{Ti}, \mathrm{K}, \mathrm{Mn}, \mathrm{Cu}, \mathrm{V}, \mathrm{Cr}, \mathrm{Rb}, \mathrm{Sr}, \mathrm{Ba}$, and $\mathrm{Pb}$. No elements from halogen group, such as $\mathrm{F}$, were detected.

The FE-SEM revealed that the size of the dust particle are mostly tens up to few hundreds micrometer, with irregular and sharp with jagged edges, forms.

\section{ACKNOWLEDGEMENT}

We would like to express our gratitude to the Brawijaya 
University, for granting the fund through "Hibah Penelitian Profesor dan Doktor", from which we were granted the fund through contract number of: 16/UN10.F09/PN/2019. Our thanks must also go to the people at the LSIH of Brawijaya University, and the Central Lab of State University of Malang, where the analysis of our samples were, mostly, done.

\section{REFERENCES}

1. Ayris and Delmelle, (2012), The immediate environmental effects of tephra emission, Bull Volcanol (2012) 74:1905-1936DOI 10.1007/s00445-012-0654-5.

(https://link.springer.com/content/pdf/10.1007/s00445-012-0654-5.pdf)

2. Colleen M. Riley, William I. Rose, and Gregg J. S. Bluth, (2003), Quantitative shape measurements of distal volcanic ash, JOURNAL OF GEOPHYSICAL RESEARCH, VOL. 108, NO. B10, 2504, doi:10.1029/2001JB000818, 2003

3. Cullen, R. T., AD Jones, BG Miller, CL Tran, JMG Davis, K Donaldson, M Wilson, V Stone, A Morgan, 2002, Toxicity of volcanic ash from Montserrat, Napier University, Edinburgh.

4. Durand, M. and J. Grattan, (2001), "Effects of volcanic air pollution on health", Lancet. 2001 Jan 20;357(9251):164

5. Horwell, Claire J and Baxter, Peter J., (2006), Bull. Volcanol. July (2006), Volume 69, Issue 1, pp 1-24. DOI 10.1007/s00445-006-0052-y

6. International Center for Diffraction Data (ICDD), http://www.icdd.com/

7. Nanzyo, Masami, (2002), Unique Properties of Volcanic as Soils, Tohoku University, Sendai, Japan

8. Omar, H. D., (2015), "To Investigation the Structure and Morphology of Iron Metallic by Difference Techniques”, J. Nano. Adv. Mat. 3, No. 2, 57-61

9. Prata, A. J. and Grant, I. F., (2001), Determination of mass loadings and plume heights of volcanic clouds from satellite data CSIRO Technical Paper 48, CSIRO Dept. of Atmospheric Science, Melbourne, Australia

10. Richards, Michael S., (2006), Volcanic Ash Cloud Height Using the MODIS CO2 Slicing Algorithm, Master Thesis, University Wisconsin

11. Sammond, Peter, McGuire, Bill, and Edwards, Stephen (eds), (2010), "Volcanic Hazard from Iceland; Analysis and Implication of the Eyjaffjallajokull Eruption" UCL Institute for Risk and Disaster Reduction, University College London

12. Thomas M.Wilson, CarolStewart, VictoriaSword-Daniels, Graham S.Leonard, David M.Johnston, Jim W.Cole, JohnnyWardman, GrantWilson, Scott T.Barnard, (2012), "Volcanic ash impacts on critical infrastructure", Physics and Chemistry of the Earth, Parts A/B/C, Volumes 45-46, 2012, Pages 5-23

13. Vogel, et al., (2016), Reference data set of volcanic ash physicochemical and optical properties, Journal of Geophysical Research: Atmospheres, Res. Article DOI: 10.1002/2016JD026328 (https://agupubs.onlinelibrary.wiley.com/doi/epdf/10.1002/2016JD0263 28)

14. Witham, C.S.; Oppenheimer, C.; Horwell, C.J. (2005). "Volcanic ash-leachates: a review and recommendations for sampling methods". Journal of Volcanology and Geothermal Research. 141 (3): 299-326

\section{AUTHORS PROFILE}

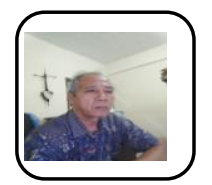

Alamsyah M. Juwono, is lecture at the Study Program of Geophysics Engineering, Deparment of Physics, Faculty of Mathematics and Sciences, Brawijaya University. He is a graduate from Gadjah Mada University, Jogjakarta, studied for Sarjana (Drs) Degree in Theoretical Physics, His master degree (M.Sc.) was obtained in the field of Cosmic Ray Astonomy, at the University of Tasmania, Hobart, Australia, and his doctoral degree $(\mathrm{PhD})$ was obtained in the Environmental Physics, at the Queensland Univeristy of Technology (QUT), Brisbane Australia. His research interest is in the environmental geophysical applications and hazard mitigation, ranging from air quality, due to diesel emission, to the impacts of volcanic ash/dust from eruption, such as to the agricultural, health, telecommunication, and transport systems. At Brawijaya University, he teaches various kinds of physics and geophysics subjects, such as mechanics and electrodynamics, Potential Field Theory, Gravity and Magnetic Survey method, Structural Geology, and some more other subjects. He is a member of Association of Indonesia Geophysicists (HAGI), a member of Indonesian Physicists Association (HFI), and a member of American Association of Petroleum Geologists (AAPG). 